\title{
Novel KCNQ2 channel activators discovered using fluorescence-based and automated patch-clamp- based high-throughput screening techniques
}

\author{
Jin-feng YUE ${ }^{1}$, Guan-hua QIAO ${ }^{1}$, Ni LIU ${ }^{2,3}$, Xue-qin $\mathrm{CHEN}^{1}$, Fa-jun NAN ${ }^{2, *}$, Zhao-bing GAO ${ }^{1, *}$ \\ ${ }^{1}$ CAS Key Laboratory of Receptor Research, ${ }^{2}$ The National Center for Drug Screening, Shanghai Institute of Materia Medica, State Key \\ Laboratory of Drug Research, Chinese Academy of Sciences, Shanghai 201203, China; ${ }^{3}$ Department of Chemistry and Institute of \\ Medicinal Chemistry, East China Normal University, Shanghai 200062, China
}

Aim: To establish an improved, high-throughput screening techniques for identifying novel KCNQ2 channel activators.

Methods: KCNQ2 channels were stably expressed in $\mathrm{CHO}$ cells (KCNQ2 cells). Thallium flux assay was used for primary screening, and 384-well automated patch-clamp lonWorks Barracuda was used for hit validation. Two validated activators were characterized using a conventional patch-clamp recording technique.

Results: From a collection of 80000 compounds, the primary screening revealed a total of 565 compounds that potentiated the fluorescence signals in thallium flux assay by more than $150 \%$. When the 565 hits were examined in lonWorks Barracuda, 38 compounds significantly enhanced the outward currents recorded in KCNQ2 cells, and were confirmed as KCNQ2 activators. In the conventional patch-clamp recordings, two validated activators ZG1732 and ZG2083 enhanced KCNQ2 currents with EC50 values of $1.04 \pm 0.18$ $\mu \mathrm{mol} / \mathrm{L}$ and $1.37 \pm 0.06 \mu \mathrm{mol} / \mathrm{L}$, respectively.

Conclusion: The combination of thallium flux assay and lonWorks Barracuda assay is an efficient high-throughput screening (HTS) route for discovering KCNQ2 activators.

Keywords: KCNQ2; channel activator; high-throughput screening; thallium flux assay; automated patch-clamp; ZG1732; ZG2083

Acta Pharmacologica Sinica (2016) 37: 105-110; doi: 10.1038/aps.2015.142

\section{Introduction}

With more than 40 members, voltage-gated potassium $(\mathrm{Kv})$ channels are a major drug target group that is essential for treating a variety of human diseases. The neuronal KCNQ2 potassium channel (Kv7.2) is a low-threshold Kv channel that is expressed in both the central and peripheral nervous systems ${ }^{[1-3]}$. As an essential player that mediates neuronal muscarinic $(\mathrm{M})$ currents and controls neuronal excitability, activating a KCNQ2 channel or heterogeneous KCNQ2 complexes will inhibit action potential initiation and, thus, dampen neuronal excitability ${ }^{[2]}$. In contrast, inhibiting the channel causes hyperexcitability ${ }^{[4-6]}$. Mutations in human KCNQ2 genes that reduce or eliminate channel activity cause a single type of familial convulsion ${ }^{[7,8]}$. Activation of the KCNQ2 channel by synthetic compounds may dampen membrane excitability

\footnotetext{
${ }^{*}$ To whom correspondence should be addressed.

E-mail fjnan@mail.shcnc.ac.cn (Fa-jun NAN); zbgao@simm.ac.cn (Zhao-bing GAO)

Received 2015-10-12 Accepted 2015-11-12
}

and, therefore, may be a strategy for treating hyperexcitability conditions, such as epilepsy and neuropathic pain ${ }^{[3]}$. In 2011, retigabine (RTG) became the first approved KCNQ activator to treat human epilepsy ${ }^{[9]}$. With the exception of retigabine, few novel KCNQ activators have been reported in the past few decades ${ }^{[8,10-16]}$. Identifying novel activators is beneficial for understanding channel activity mechanisms and developing new therapeutics. For example, one study used ztz240 as a chemical probe to demonstrate that the KCNQ2 channel voltage-sensing domains are novel anti-epilepsy targets ${ }^{[17]}$. Benzbromarone (BBR) is a KCNQ activator that primarily acts upon peripheral KCNQ channels. The unique tissue distribution of BBR allowed scientists to resolve a long-standing debate concerning the central or peripheral mediation of analgesic activity of KCNQ activators. This provided a new strategy for developing more effective pain therapies ${ }^{[10]}$.

To discover novel small molecule activators for KCNQ2 channels from large compound libraries, various highthroughput screening (HTS) techniques have been developed. Fluorescence-based assays provide robust and homogeneous 
cell population measurements and have been widely used for both cation and anion-permeable channels. $\mathrm{K}^{+}$channels are permeable to $\mathrm{Tl}^{+[18]}$. The $\mathrm{Tl}^{+}$indicator FluxOR has been used in conjunction with a fluorescent reader for the high-throughput detection of intracellular $\mathrm{Tl}^{+}$concentration changes. The thallium flux assay has been used to identify $\mathrm{K}^{+}$channel activators in multiple large-scale screening experiments ${ }^{[19-21]}$. Electrophysiological recording directly provides ionic currents and is considered to be a gold standard for studying ion channels. Nevertheless, due to their low throughput, conventional patch-clamp experiments are not appropriate for large-scale screening. Over the past few decades, many automated electrophysiology platforms with highly improved throughput have been developed. Therefore, directly measuring ionic currents in HTS is a reality ${ }^{[22-24]}$. The IonWorks Barracuda is a 384well format automated patch that relies on a perforated patchclamp technique. Compared with earlier generations of this instrument, the IonWorks Barracuda provides better throughput and data consistency; however, the feasibility of using the IonWorks Barracuda in a KCNQ2 study has not been reported. Herein, we screened a collection of 80000 compounds using the thallium flux assay as the primary screen and then used the IonWorks Barracuda for a secondary validation. Two validated hits were further characterized using a conventional patch clamp, which revealed an $\mathrm{EC}_{50}$ of 1-2 $\mu \mathrm{mol} / \mathrm{L}$. Our study provides an efficient HTS means for identifying novel KCNQ2 activators (Figure 1).

\section{Materials and methods}

\section{Thallium flux assay}

Chinese hamster ovary ( $\mathrm{CHO}$ ) cells that stably expressed KCNQ2 channels were routinely cultured in F-12 medium supplemented with 10\% FBS and $250 \mu \mathrm{g} / \mathrm{mL}$ Hygromycin B. KCNQ2 cells were seeded into a 384-well black clear bottom plate at a concentration of 8000 cells/well by a multidrop dispenser, and the plate was incubated overnight at $37^{\circ} \mathrm{C}$ in a $5 \% \mathrm{CO}_{2}$ incubator. The next day, after manual removal of the medium, $25 \mu \mathrm{L} /$ well dye-loading buffer $(1000 \times$ FluxOR

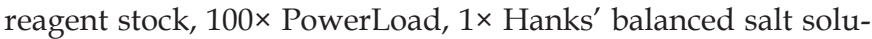
tion, $20 \mathrm{mmol} / \mathrm{L}$ HEPES, and $2.5 \mathrm{mmol} / \mathrm{L}$ Probenecid, $\mathrm{pH}$ 7.40) was added into the cell plate, and the plate was then incubated in the dark at room temperature for $90 \mathrm{~min}$. Once the dye-loading buffer was manually removed, the cell plates were then supplemented with $20 \mu \mathrm{L} /$ well assay buffer $(1 \times$ Hank's balanced salt solution, $20 \mathrm{mmol} / \mathrm{L}$ HEPES, and 2.5 mmol/L Probenecid, pH 7.40). Additionally, $4 \mu \mathrm{L} /$ well compound buffer was added into the plate, and the plate was then incubated at room temperature in the dark. After the tested compounds were incubated for $30 \mathrm{~min}$, cell plates were loaded onto an FDSS (Hamamatsu Photonics). After $10 \mathrm{~s}$ of recording, $6 \mu \mathrm{L} /$ well of stimulus buffer was added. The plates were read every second for $180 \mathrm{~s}$. The stimulus buffer contained 25 $\mathrm{mmol} / \mathrm{L} \mathrm{K}^{+}$and $25 \mathrm{mmol} / \mathrm{L} \mathrm{Tl}^{+}$. The FluxOR thallium assay protocol that has been described is identical to the manufacturer's protocol. The fluorescence potentiation (Fluo Potenti $\%=\left[\left(R_{\text {test }}-R_{\text {control }}\right) /\left(R_{\text {control }}-R_{\text {buffer }}\right) \times 100\right]$ was calculated for each well using the $35 \mathrm{~s}$ fluorescence ratio. To evaluate the quality of the thallium flux data, the Z-factor was calculated using the following equation: $Z=1-3\left(S_{c}+S D_{n}\right) /|C-N|$, where $S_{c}$ and $\mathrm{SD}_{\mathrm{n}}$ are the standard deviation of the control group $(c)$ and the group in the presence of positive control group (n), respectively. $C$ and $N$ are the mean values of the control and positive control groups, respectively.

\section{Automated patch-clamp recording}

KCNQ2 cells were dissociated from T175 flasks by aspirating off the culture medium, rinsing the cells twice with phosphate buffered saline solution (PBS, Invitrogen) and adding $3 \mathrm{~mL}$ trypsin-EDTA per flask. The cells were incubated for $3 \mathrm{~min}$ in a $37^{\circ} \mathrm{C}$ incubator. The flasks were added to $7 \mathrm{~mL}$ of cell culture medium, and the cells were gently triturated by pipetting up and down two to three times. Cells were pelleted at 800 rounds per minute for $3 \mathrm{~min}$. The supernatant was discarded and the cell pellet was resuspended in $5 \mathrm{~mL}$ of external solution at a density of 1.5-2.0 million cells per mL. The 384well automated patch clamp IonWorks Barracuda (Molecular Devices) in the population patch clamp (PPC) mode was used for hit validation. The cells were voltage clamped at a holding potential of $-100 \mathrm{mV}$. The KCNQ2 current was activated by depolarizing to $-10 \mathrm{mV}$ for $10 \mathrm{~s}$, after which the voltage was taken back to $-120 \mathrm{mV}$ for $10 \mathrm{~s}$ to observe the deactivating tail current. The maximum amount of outward current size was

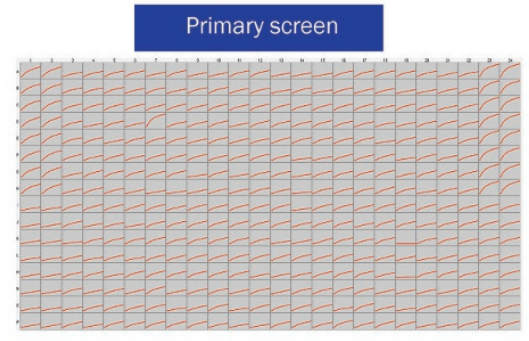

Thallium flux assay

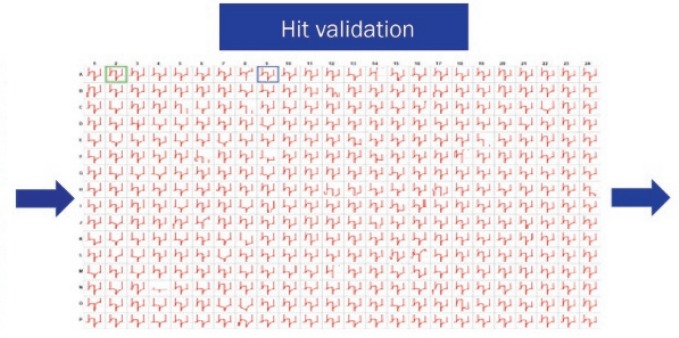

384-well lonWorks barracuda
Probe characterization

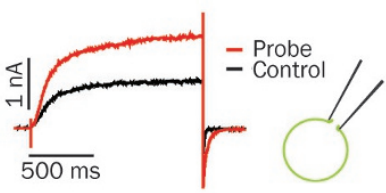

Conventional patch clamp

Figure 1. Flow-chart of the high-throughput screening route for identifying KCNQ2 activators. The fluorescence-based thallium flux assay was used for the primary screen, followed by the confirmatory 384-well lonWorks Barracuda for hit validation. After the hit validation, a conventional patch-clamp experiment was used for probe characterization. 
used to determine KCNQ2 current amplitude.

\section{Conventional patch-clamp recording}

Standard whole-cell recording was used to record the current of KCNQ2 channels. Pipettes were pulled from borosilicate glass capillaries (TW150-4, World Precision Instruments, Sarasota, FL, USA). When filled with the intracellular solution, the pipettes had a 3-5 M $\Omega$ resistance. During the recording, the extracellular solution was constantly perfused by a BPS perfusion system (ALA Scientific Instruments). The intracellular solution contained (in mmol/L): $140 \mathrm{KCl}, 1.75 \mathrm{MgCl}_{2}, 10$ EGTA, 10 HEPES and $5 \mathrm{Na}_{2} \mathrm{ATP}(\mathrm{pH}=7.2)$; the extracellular solution contained (in mmol/L): $145 \mathrm{NaCl}, 3 \mathrm{KCl}, 2 \mathrm{CaCl}_{2}, 1$ $\mathrm{MgCl}_{2}, 10 \mathrm{HEPES}$ and 10 glucose $(\mathrm{pH}=7.40)$. Both currents and voltages were recorded using an Axopatch-200B amplifier, filtered at $2 \mathrm{kHz}$, and digitized using a DigiData 1440A with pClamp 10.2 software (Molecular Devices). Series resistance compensation was also used and set to $60 \%-80 \%$. Patch clamp data were processed using Clampfit 10.2 (Molecular Devices, Sunnyvale, CA, USA) and then analyzed in GraphPad Prism 5 (GraphPad Software). Voltage-dependent activation curves were fitted using the Boltzmann equation, $G=G_{\min }+\left(G_{\max }-\right.$ $\left.G_{\min }\right) /\left(1+\exp \left(V-V_{1 / 2}\right) / S\right)$, where $G_{\max }$ is the maximum conductance, $G_{\min }$ is the minimum conductance, $V_{1 / 2}$ is the voltage for reaching $50 \%$ of maximum conductance, and $S$ is the slope factor. Dose-response curves were fitted with the Hill equation, $E=E_{\max } /\left(1+\left(E_{50} / C\right) P\right)$, where $E_{50}$ is the drug concentration that produced half of the maximum response, and $P$ is the Hill coefficient.

\section{Statistical analysis}

The data are presented as the mean \pm SEM. The significance was estimated using paired two-tailed Student's t-tests. An effect was considered significant if $P<0.05$.

\section{Results}

\section{Primary screening using the thallium flux assay}

To identify compounds with potentiation effects on KCNQ2 channels, we generated a KCNQ2-stable cell line. After a series of optimization experiments for several important screening parameters, which have been described previously, a standard procedure for the thallium flux assay was developed and used to screen an in-house collection of over 80000 compounds at a $10 \mu \mathrm{mol} / \mathrm{L}$ final concentration (Figure 2). The positive control was ztz240, which is a KCNQ2 channel activator that was previously identified to significantly increase the fluorescence signal by approximately $150 \%$ at $10 \mu \mathrm{mol} / \mathrm{L}$. Of the 80000 screened compounds, 565 hits were discovered that exhibited a larger potentiation activity than ztz240, as exemplified by ZG1732. The $Z$ factors ranged from 0.51 to 0.89 , which are consistent with the assay quality requirement of being greater than 0.5 .

\section{Hit validation using an automated patch-clamp experiment}

A 384-well automated patch-clamp instrument, the IonWorks Barracuda, was used to validate the hits. The stabilities of the
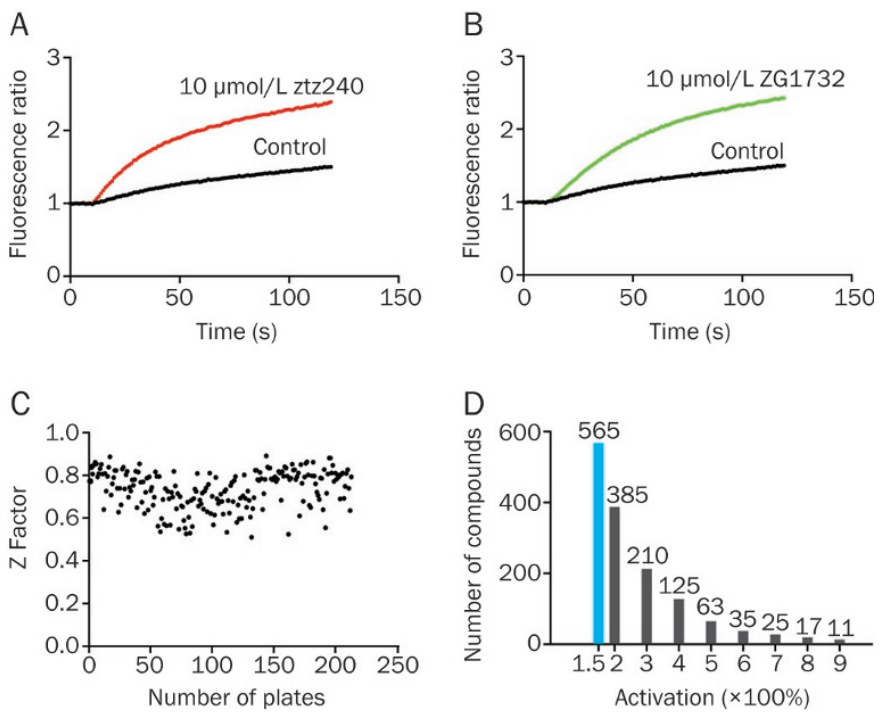

Figure 2. Results of thallium flux assay. (A and B) Representative fluorescence traces in the absence or presence of $10 \mu \mathrm{mol} / \mathrm{L}$ ztz240 (A) or $10 \mu \mathrm{mol} / \mathrm{L}$ ZG1732 (B). (C) Z factors of the thallium flux assay. (D) Distribution of identified hits in a total of 80000 compounds in the thallium flux assay. The $x$-axis represents the potentiation activity of the hits on the KCNQ2-mediated fluorescence signal (see the Methods for the calculation method).

KCNQ2 currents were evaluated first. The cells were held at $-100 \mathrm{mV}$ and stimulated by depolarization to $-10 \mathrm{mV}$ every 20 s. Prior to each depolarization event, a 20-ms pre-pulse that polarizes the membrane potential to $-90 \mathrm{mV}$ (a "sweep") was delivered for leak subtraction. Under these conditions, the outward currents of the KCNQ2 channels remained relatively stable for $5 \mathrm{~min}$. We then examined the KCNQ2 current responses to $10 \mu \mathrm{mol} / \mathrm{L}$ ztz240. As shown in Figure 3, the drug was applied after the 3rd sweep. The increased KCNQ2 currents reached a steady state by the 5th sweep, and a slight run-down was observed at the 7 th sweep. Therefore, the currents measured at the 6th sweep were used to determine the potentiation activity of the tested hits. Of the 565 hits that were discovered in the primary screen, 38 were confirmed as KCNQ2 channel activators (Figure 3C and 3D, and Supplementary Table S1). Most of the validated activators displayed comparable potentiation activity to ztz240, which is exemplified by ZG1732.

\section{Characterization of activators using conventional patch clamp}

Among the 38 validated activators, ZG1732 and ZG2083, which belong to different scaffolds, were selected for further characterization using a conventional whole-cell patch-clamp, the gold standard for studying ion channels. Similar to the KCNQ2 activators that were identified previously, including retigabine and ztz240, both ZG1732 and ZG2083 increased the outward KCNQ2 currents and shifted the activation curve to the left (Figure 4). The $\mathrm{EC}_{50}$ values of ZG1732 and ZG2083 were $1.04 \pm 0.18 \mu \mathrm{mol} / \mathrm{L}$ and $1.37 \pm 0.06 \mu \mathrm{mol} / \mathrm{L}$, respectively. The micromolar $\mathrm{EC}_{50}$ values are comparable to the hits that 
A
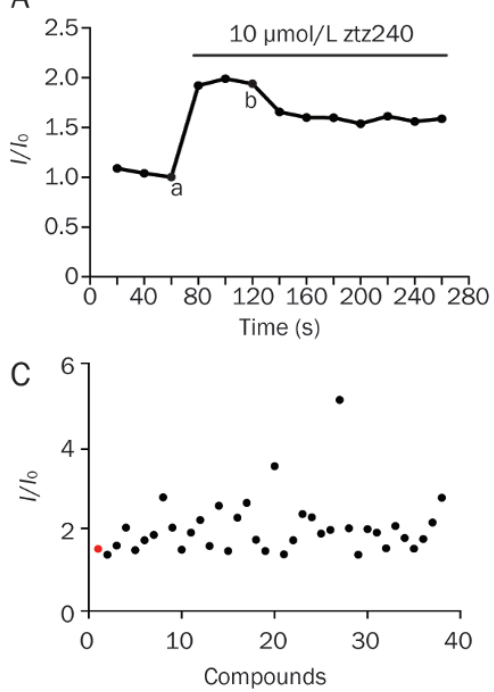

B $\quad-10 \mu \mathrm{mol} / \mathrm{L} \mathrm{ztz} 240$

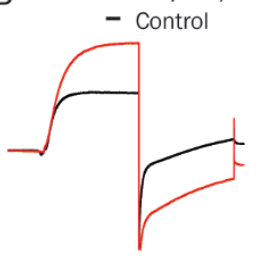

$\sqrt[-100 \mathrm{mV}]{-110 \mathrm{mV}}$

D

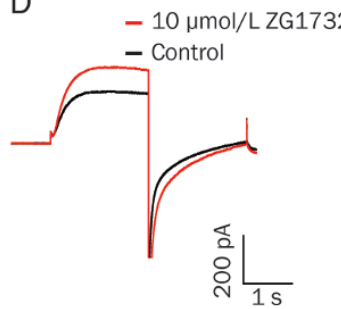

Figure 3. Results of IonWorks Barracuda assay. (A) Stability of KCNQ2 currents that were recorded using the lonWorks Barracuda instrument. ztz240 (10 $\mu \mathrm{mol} / \mathrm{L})$ was applied at the 3rd sweep (a). The increased KCNQ2 current was measured before the 'run-down' appeared (the 6th sweep (b). (B) Representative KCNQ2 currents before and after exposure to $10 \mu \mathrm{mol} / \mathrm{L}$ ztz240. (C) Potentiation of the validated hits on the KCNQ2 currents. (D) Representative KCNQ2 currents before and after exposure to $10 \mu \mathrm{mol} / \mathrm{L}$ ZG1732 [red point in (C)].

were identified in a previous HTS ${ }^{[25]}$.

\section{Discussion}

In the current study, the thallium flux assay was used as the

primary screening method, and the 384-well IonWorks Barracuda instrument was used for the secondary validation. Currently, the thallium flux assay remains the prevalent HTS method for identifying potassium channel modulators, including KCNQ channels ${ }^{[19,25]}$. The thallium assay can be used to identify both activators and inhibitors of KCNQ channels. Typically, the hits identified in a primary screen require further chemical modification to improve potency and druggability. For example, a potent KCNQ1 activator was developed based upon an initial hit that was identified in an HTS of the Molecular Libraries Probe Centers Network (MLPCN) library using the thallium assay ${ }^{[26]}$. In another HTS by the same group, a potent activator of KCNQ2 channels was designed and synthesized based upon the identified hits ${ }^{[25]}$; however, due to the off-target effects and autofluorescence of the compounds, a high false positive hit rate is a major issue for this indirect fluorescence assay. In a screen for KCNQ4 activators, the false positive rate was greater than $93 \%(623 / 666)$ in the primary screening stage ${ }^{[21]}$. Consistent with this previous study, the false positive rate for our thallium assay was also $93 \%(527 / 565)$. To reduce the false positive rate, a secondary confirmation is often required with duplicates for each compound and counter screening for the parental cells that do not express the KCNQ channels investigated ${ }^{[21]}$. A conventional patch-clamp experiment is the gold standard for studying ion channels and provides a large quantity of information as well as high resolution; however, due to intrinsic limitations, particularly due to its low throughput, conventional patchclamp is not able to satisfy the need of high-throughput drug discovery ${ }^{[27]}$. In response to the demand for high-throughput experiments, many automated patch-clamp systems have been
A
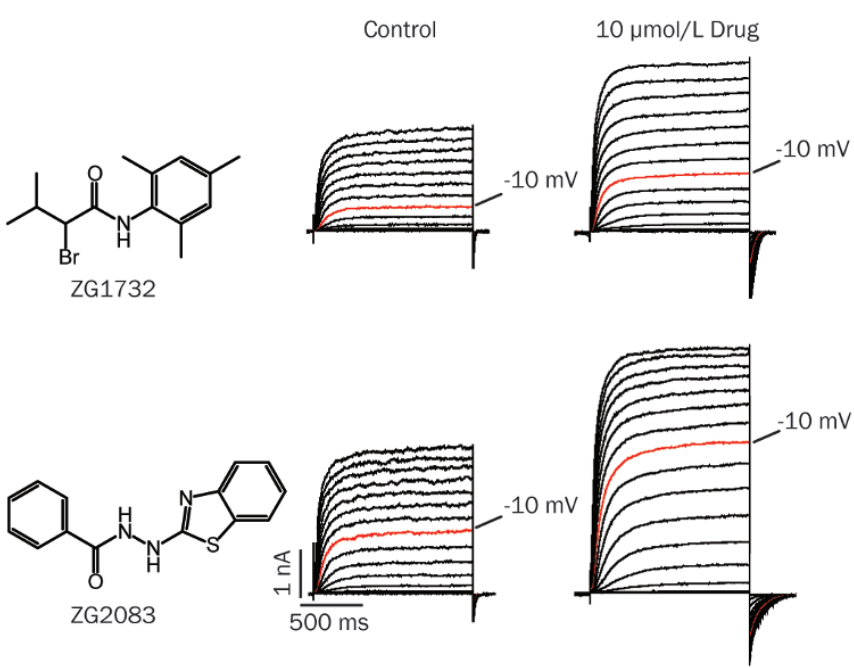

B
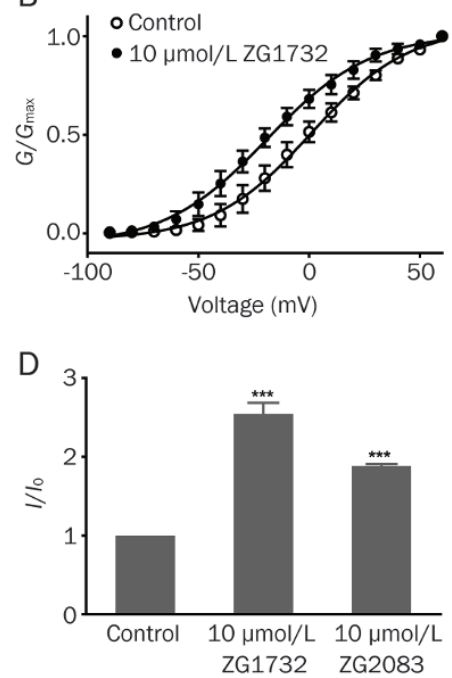

$c$

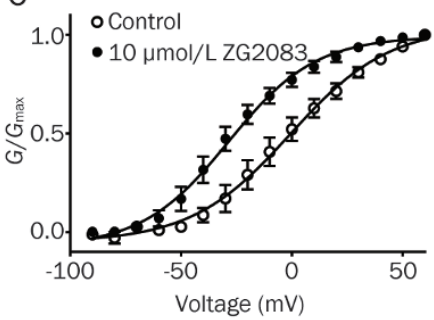

$\mathrm{E}$

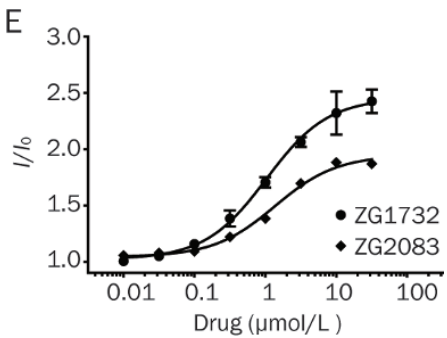

Figure 4. Potentiation effects of the hits ZG1732 and ZG2083 on KCNQ2 channels using a conventional patch-clamp. (A) Representative currents of KCNQ2 channels in the absence or presence of indicated hits. To elicit the KCNQ2 currents, depolarization steps that ranged from $-90 \mathrm{mV}$ to +60 $\mathrm{mV}$ in $10 \mathrm{mV}$ increments were applied from the holding potential of -100 mV. (B and C) ZG1732 and ZG2083 left-shifted the conductance-voltage (GV) curve of KCNQ2 channels. For ZG1732, $\Delta V_{1 / 2}=-19.88 \pm 0.12 \mathrm{mV}(n=5)$; for ZG2083, $\Delta V_{1 / 2}=-27.79 \pm 0.73 \mathrm{mV}$ ( $\left.n=4\right)$. (D) Potentiation activity of 10 $\mu \mathrm{mol} / \mathrm{L} Z \mathrm{ZG} 1732\left(\mathrm{I} / \mathrm{I}_{0}=2.54 \pm 0.14, n=4\right)$ and $10 \mu \mathrm{mol} / \mathrm{L} Z \mathrm{G} 2083\left(\mathrm{I} / \mathrm{I}_{0}=1.88 \pm 0.02, n=7\right)$ on the KCNQ2 current amplitude measured at $-10 \mathrm{mV}$. (E) Doseresponse curves of ZG1732 and ZG2083 $(n=5)$. Mean \pm SEM. ${ }^{c} P<0.01$. 
developed throughout the last 30 years ${ }^{[28]}$. These automated patch-clamp systems can be divided into automated conventional electrophysiology platforms that use glass pipettes and automated planar-array electrophysiology platforms. Nevertheless, only the latter have overcome the throughput limitations and were first used for screening in $2003^{[29]}$. The IonWorks Barracuda is a 384-well planar-array platform that can simultaneously record 384 currents. As a high-throughput patch-clamp instrument that is specifically designed for highthroughput drug discovery, the utility of IonWorks Barracuda must be tested and validated for various ion channels. In the current study, the IonWorks Barracuda can stably record KCNQ2 currents for up to 5 min; thus, it is feasible to evaluate KCNQ2 activators that typically reach steady potentiation within a few minutes. The validated activators were further characterized using conventional patch clamp studies, which confirmed the IonWorks Barracuda results. In summary, our study established an efficient HTS means for identifying KCNQ2 activators, which can be referred to or is applicable for other voltage-gated potassium channels.

\section{Acknowledgements}

We gratefully acknowledge the financial support from the National Natural Science Foundation of China Grant for Excellent Key Laboratory (81123004), the National Natural Science Foundation of China (61327014, 61175103 and 91413122), Shanghai Municipal Science and Technology Commission (13JC1406700) and the External Cooperation Program of BIC, Chinese Academy of Sciences (1536631KYSB20130003).

\section{Author contribution}

Zhao-bing GAO and Fa-Jun NAN designed the project; Jinfeng YUE, Guan-hua QIAO, Ni LIU, and Xue-qin CHEN performed the experiments; all authors analyzed the data; Jinfeng YUE and Zhao-bing GAO wrote the manuscript.

\section{Supplementary information}

Supplementary Table S1 is available at Acta Pharmacologica Sinica's website.

\section{References}

1 Brown DA, Adams PR. Muscarinic suppression of a novel voltagesensitive $\mathrm{K}^{+}$current in a vertebrate neurone. Nature 1980; 283: 673-6.

2 Robbins J. KCNQ potassium channels: physiology, pathophysiology, and pharmacology. Pharmacol Ther 2001; 90: 1-19.

3 Xiong Q, Gao Z, Wang W, Li M. Activation of Kv7 (KCNQ) voltage-gated potassium channels by synthetic compounds. Trends Pharmacol Sci 2008; 29: 99-107.

4 Brown DA, Passmore GM. Neural KCNQ (Kv7) channels. Br J Pharmacol 2009; 156: 1185-95.

5 Piccinin S, Randall AD, Brown JT. KCNQ/Kv7 channel regulation of hippocampal gamma-frequency firing in the absence of synaptic transmission. J Neurophysiol 2006; 95: 3105-12.

6 Yue C, Yaari Y. KCNQ/M channels control spike afterdepolarization and burst generation in hippocampal neurons. J Neurosci 2004; 24: 4614-24.

7 Charlier C, Singh NA, Ryan SG, Lewis TB, Reus BE, Leach RJ, et al.
A pore mutation in a novel KQT-like potassium channel gene in an idiopathic epilepsy family. Nat Genet 1998; 18: 53-5.

8 Singh NA, Charlier C, Stauffer D, DuPont BR, Leach RJ, Melis R, et al. A novel potassium channel gene, KCNQ2, is mutated in an inherited epilepsy of newborns. Nat Genet 1998; 18: 25-9.

9 Tatulian L, Delmas P, Abogadie FC, Brown DA. Activation of expressed KCNQ potassium currents and native neuronal M-type potassium currents by the anti-convulsant drug retigabine. J Neurosci 2001; 21: 5535-45.

10 Zheng Y, Xu H, Zhan L, Zhou X, Chen X, Gao Z. Activation of peripheral KCNQ channels relieves gout pain. Pain 2015; 156: 1025-35.

11 Bentzen BH, Schmitt N, Calloe K, Dalby Brown W, Grunnet M, Olesen SP. The acrylamide (S)-1 differentially affects Kv7 (KCNQ) potassium channels. Neuropharmacology 2006; 51: 1068-77.

12 BlomSM, Schmitt N, Jensen HS. The acrylamide (S)-2 as a positive and negative modulator of $\mathrm{Kv} 7$ channels expressed in Xenopus laevis oocytes. PLoS One 2009; 4: e8251.

13 Fritch PC, McNaughton-Smith G, Amato GS, Burns JF, Eargle CW, Roeloffs R, et al. Novel KCNQ2/Q3 agonists as potential therapeutics for epilepsy and neuropathic pain. J Med Chem 2011; 53: 887-96.

14 Jia C, Qi J, Zhang F, Mi Y, Zhang X, Chen X, et al. Activation of KCNQ2/3 potassium channels by novel pyrazolo[1,5-a]pyrimidin7(4H)-one derivatives. Pharmacology 2011; 87: 297-310.

15 Peretz A, Pell L, Gofman Y, Haitin Y, Shamgar L, Patrich E, et al. Targeting the voltage sensor of Kv7.2 voltage-gated $\mathrm{K}^{+}$channels with a new gating-modifier. Proc Natl Acad Sci U S A 2010; 107: 15637-42.

16 Wickenden AD, Krajewski JL, London B, Wagoner PK, Wilson WA, Clark S, et al. N-(6-chloro-pyridin-3-yl)-3,4-difluoro-benzamide (ICA27243): a novel, selective KCNQ2/Q3 potassium channel activator. Mol Pharmacol 2008; 73: 977-86.

17 Li P, Chen Z, Xu H, Sun H, Li H, Liu H, et al. The gating charge pathway of an epilepsy-associated potassium channel accommodates chemical ligands. Cell Res 2013; 23: 1106-18.

18 Wagoner PK, Oxford GS. Cation permeation through the voltagedependent potassium channel in the squid axon. Characteristics and mechanisms. J Gen Physiol 1987; 90: 261-90.

19 Raphemot R, Kadakia RJ, Olsen ML, Banerjee S, Days E, Smith SS, et al. Development and validation of fluorescence-based and automated patch clamp-based functional assays for the inward rectifier potassium channel Kir4.1. Assay Drug Dev Technol 2013; 11: 532-43.

20 Yu H, Xu K, Zou B, Wu M, McManus OB, Le Engers J, et al. Identification of a novel, small molecule inhibitor of KCNQ2 channels. Probe Reports from the NIH Molecular Libraries Program [Internet]. Bethesda (MD): National Center for Biotechnology Information (US) 2010-2011 Oct 28.

21 Li Q, Rottlander M, Xu M, Christoffersen CT, Frederiksen K, Wang MW, et al. Identification of novel KCNQ4 openers by a high-throughput fluorescence-based thallium flux assay. Anal Biochem 2011; 418: 66-72.

22 Ion channel screening - automated patch clamp on the rise. Drug Discov Today Technol 2008; 5: e1-e34.

23 Lepple-Wienhues A, Lassen D, Hummer A, Czubayko U, Knirsch M, Golubovic A, et al. The Chiptip: a novel tool for automated patch clamp. Comb Chem High Throughput Screen 2009; 12: 73-7.

24 Shieh CC. Automated high-throughput patch-clamp techniques. Drug Discov Today 2004; 9: 551-2.

25 Yu H, Wu M, Hopkins C, Engers J, Townsend S, Lindsley C, et al. A small molecule activator of KCNQ2 and KCNQ4 channels. Probe Reports from the NIH Molecular Libraries Program [Internet]. Bethesda (MD): National Center for Biotechnology Information (US) 2010-2011 Mar 29.

26 Mattmann ME, Yu H, Lin Z, Xu K, Huang X, Long S, et al. Identification of (R)-N-(4-(4-methoxyphenyl)thiazol-2-yl)-1-tosylpiperidine-2- 
carboxamide, ML277, as a novel, potent and selective K(v)7.1 (KCNQ1) potassium channel activator. Bioorg Med Chem Lett 2012; 22 : 5936-41.

27 Py C, Martina M, Diaz-Quijada GA, Luk CC, Martinez D, Denhoff MW, et al. From understanding cellular function to novel drug discovery: the role of planar patch-clamp array chip technology. Front Pharmacol 2011; 2: 51.
28 Carmelle V. Conventional Patch Clamp Techniques and HighThroughput Patch Clamp Recordings on a Chip for Measuring Ion Channel Activity, in Textbook of Pulmonary Vascular Disease 2010; Springer. USA. P 495-510.

29 Schroeder K, Neagle B, Trezise DJ, Worley J. lonworks HT: a new highthroughput electrophysiology measurement platform. J Biomol Screen 2003; 8: 50-64. 